\title{
Metodología para la planificación del entrenamiento en el tenis de alta competición
}

\section{RESUMEN}

El presente artículo ofrece al lector una visión del proceso de entrenamiento en la que los aspectos técnicos, tácticos, físicos y psicológicos puedan integrarse, con el objeto de aportar la más elevada productividad a cada sesión de entrenamiento en el tenis de alta competición. Así, mediante este trabajo pretendemos mostrar las nuevas posibilidades que el denominado entrenamiento integrado ofrece al desarrollo de la programación de las sesiones de entrenamiento, con el fin de abrir nuevas vías por lo que a la contemplación de la periodización y planificación del entrenamiento del tenis de alta competición se refiere.

Tras la introducción, que recoge los puntos de vista de varios autores acerca de las bases más recientes sobre las que ha venido sustentándose el entrenamiento deportivo, sigue la exposición de diversos aspectos que caracterizan la planificación actual del entrenamiento en el tenis de alta competición, así como una serie de referencias dedicadas a la naturaleza del trabajo en equipo multidisciplinar, amén de otro apartado, el cual tiene como núcleo temático la metodología para el diseño de las sesiones de entrenamiento integrado. Un ejemplo de ejercicio técnico-táctico correspondiente a una sesión de entrenamiento integrado en el tenis cierra el artículo.

Palabras clave: planificación, trabajo multidisciplinar, alta competición, tenis.
Juan Pedro Fuentes García Universidad de Extremadura, Cáceres (España)

Fernando Del Villar Alvarez Universidad de Extremadura, Cáceres (España)

Luis Antonio Ramos Mondejar Universidad de Extremadura, Cáceres (España) Perla Moreno Arroyo Universidad de Extremadura, Cáceres (España)

\section{INTRODUCCIÓN}

Schonborn (1983) apunta las líneas que han venido caracterizando al entrenamiento deportivo hasta fechas muy recientes, a saber:

- Excesivo número de jugadores.

- Sesiones demasiado breves (circunstancia que imposibilita su división en fases).

- Inexistencia de planificación (lo que implica la ausencia de fases).

- La no contemplación de la edad física y del entrenamiento.

- Una dedicación excesiva a los aspectos técnicos. - La no consideración de la influencia de los factores limitadores y potenciadores del rendimiento.

- La sobrevaloración de los resultados obtenidos en los torneos (especialmente en el caso de los jugadores más jóvenes).

Las consecuencias de este tipo de entrenamiento se hacen más que notorias: de un lado, la ausencia de una evolución progresiva en etapas predeterminadas (como mucho, la progresión es lineal), extremo este del todo incompatible con el nivel de exigencia que define al deporte actual; de otro, la no consecución por parte del jugador de su límite individual de rendimiento hasta una etapa posterior.

Respecto de una de las estrategias más eficaces para el logro de rendimientos óptimos en la práctica deportiva del tenis, el entrenamiento modelado, Stojan (1988) se expresa en los siguientes términos: 


significa simular la atmósfera del partido y sus
actuaciones más comunes e importantes durante e
entrenamiento. En el sentido más amplio de
término, la práctica de cualquier golpe, táctica y
cualidad física está incluida dentro del entrenamiento
modelado. Por ejemplo, para preparar un partido
sobre pista rápida es mejor entrenar en especial e
servicio, el resto, la volea, el remate y el passing
shot. Los golpes de fondo son los menos
importantes".
En este sentido, Rasicci (1986) se refiere a algunos
aspectos que deben ser considerados a la hora de
proceder al planteamiento y desarrollo de un
entrenamiento modelado en el tenis:
- El calentamiento.
- Tipos de esfuerzos.
- Ejercicios de control y consistencia.
- Importancia de la recuperación.
- Trabajo del entrenador.
- Correcciones.
- Duración del entrenamiento y período de
temporada.
- Superficie de la pista.
- Entrenamiento real y contenidos de los
- Importancia de la competición.
ejes.

Schõnborn (1989) considera también que debe entrenarse de la manera lo más parecida posible a un partido de tenis, sin obviar el hecho, más que notable, de que hay que entrenar no únicamente en la fase anaeróbica sino también en la aeróbica, de modo que al llevar a cabo la periodización del plan de entrenamiento, deberá ponerse especial énfasis al principio a la fase aeróbica o de entrenamiento de la condición física, dedicando una mayor atención al final al entrenamiento de la técnica y las situaciones de partido. Expone el autor citado una serie de reglas para el planteamiento correcto de un entrenamiento moderno en tenis:

1. a) Establecer unos objetivos claros y exactos para cada día, unidad o ejercicio de entrenamiento.

2. $\left.{ }^{a}\right)$ Dosificar la intensidad de entrenamiento y de la fase de regeneración (peloteo de 10 segundos y pausa de 20 segundos).

3. ) Controlar la combinación y la secuencia de los diversos tipos de ejercicios, programados en orden creciente de dificultad (del trabajo de coordinación al trabajo de potencia, del trabajo técnico al trabajo táctico, etc.).

4. $\left.{ }^{\mathrm{a}}\right)$ Controlar el número de repeticiones necesarias para el aprendizaje de un determinado gesto (investigaciones en tenis han venido a confirmar que el éxito mayor del aprendizaje gira aproximadamente alrededor de las 150 repeticiones, precisando Schönborn que los buenos jugadores de alto nivel alcanzan su mejor rendimiento aproximadamente a los 100 golpes, debido a la realización por parte de los mismos de una serie de pausas que les permiten la regeneración y el rendimiento al máximo de sus posibilidades).

\section{LA PLANIFICACIÓN ACTUAL DEL ENTRENAMIENTO EN EL TENIS DE ALTA COMPETICIÓN}

La mayor parte de los autores considera que el desarrollo del trabajo de índole táctica debe venir precedido por un tipo de entrenamiento dirigido mayoritariamente a la mejora de la condición física y de los fundamentos técnicos. Para dichos autores, el período preparatorio general se estructura mediante la incorporación de tareas que conllevan una elevada carga de trabajo técnico, mientras que el período precompetitivo se caracteriza por un tipo de entrenamiento en el que se plantean situaciones de juego real modificadas.

Asimismo, se le concede escaso valor en todos los períodos de la temporada (preparatorio general, precompetitivo y competitivo) a la preparación psicológica.

Coincidimos plenamente con la opinión de Balaguer (1996), para quien "Durante un partido un jugador necesita estar preparado a muchos niveles". "Si los jugadores -prosigue el citado autor- consiguen armonizar los diferentes aspectos de su entrenamiento y entienden que en su funcionamiento no existe separadamente lo físico de lo técnico, ni de lo táctico, ni de lo psicológico, sino que es la acción conjunta de todos estos 
aspectos lo que les permite que se proyecten como un todo integrado, están en el camino de : desarrollarse y actuar al cien por cien de sus : posibilidades" (figura 1).

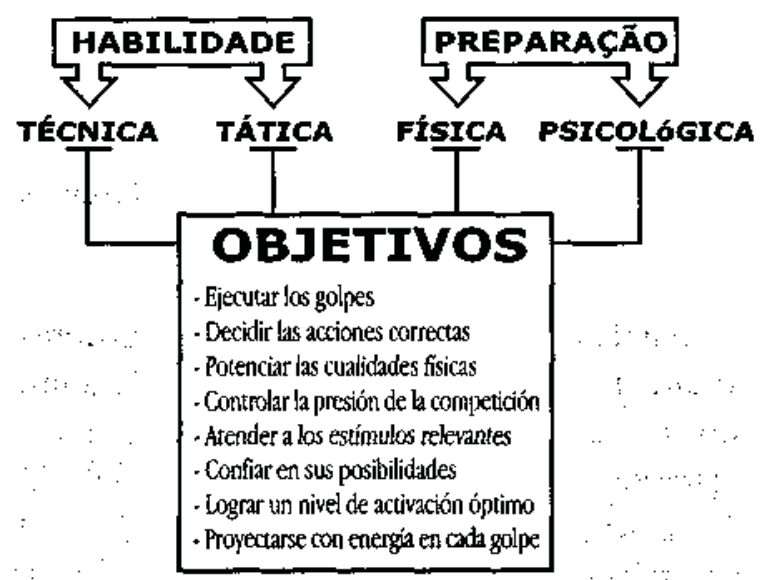

FIG. 1. Niveles de preparación del jugador de tenis. Adaptado de Balaguer (1996)

Enlazando con las ideas recogidas en el párrafo anterior, entendemos que el entrenamiento técnico, táctico, físico y psicológico debe producirse de forma paralela, con objeto de que el jugador inicie la fase de competición debidamente formado y preparado desde un punto de vista global, facilitándosele con ello la posibilidad de enfrentarse a la situación real de juego con una mayor naturalidad.

Evidentemente, para llevar a buen puerto el : entrenamiento integrado, resulta necesario sensibilizar : y formar a los entrenadores y jugadores en el sentido : de tratar de trasladar el conocimiento científico a su formación, haciéndoles ver el beneficio de desplegar : y desarrollar en el terreno de la práctica estudios científicos.

Loth (1984) propone "Cuatro horas de entrenamiento de tenis por día. El entrenamiento físico, de 1 a 1 1/2 $\mathrm{h}$ por día, sólo se organiza durante los periodos en que no está prevista ninguna competición en breve plazo".

Alvaro Alcalde (1992) sostiene que "La planificación es un proceso metodológico de estructuración de las cargas de entrenamiento, en busca del rendimiento óptimo del deportista. El deportista es considerado desde una perspectiva global e integradora de todas las facetas personales y deportivas".

Antón (1987, citado por García Herrero, 1997/1998), respecto de la escasa transferencia a la situación real de partido de que adolecían los entrenamientos, señala que "Se empezaron a plantear entrenamientos que respetaran las características del juego e intentaran desarrollar las capacidades de los jugadores, de tal forma que la transferencia al juego estuviera facilitada. Así, se comenzó a formular una nueva interpretación de los datos y valores obtenidos en los partidos, entendiendo que los valores absolutos eran poco significativos, y que posiblemente, esos parámetros había que juzgarlos desde la perspectiva del contexto y exigencias del juego".

Si durante el período preparatorio general y precompetitivo la evolución física, técnica, táctica y psicológica se produce de forma paralela, el jugador inicia el período competitivo formado desde un punto de vista global que le permite enfrentarse a una situación real de juego con mayor naturalidad, amén de rentabilizar al máximo el tiempo dedicado al entrenamiento.

Innovadora y de indudable interés resulta la propuesta de Navarro (1998), quien se refiere al "macrociclo integrado", señalando que este tipo de estructuración, que debe su nombre al hecho de que la integración de los diferentes contenidos de entrenamiento se efectúa en períodos que comprenden entre seis y diez semanas, "Agrupa los contenidos y medios de entrenamiento en corto espacio de tiempo con aplicación de éstos en forma de carga acentuada". El planteamiento de Navarro nace con la pretensión de que "el deportista tenga todos los contenidos de entrenamiento que se utilizan a lo largo de la temporada, concentrados en períodos más cortos pero suficientes para que se produzcan cambios fisiológicos adaptativos", quedando la estructura del macrociclo integrado articulada en tres fases: "fase general" (centrada en el volumen), "fase específica" (orientada a la intensidad) y "fase de mantenimiento" (destinada a producir la supercompensación del trabajo efectuado en las semanas precedentes), con, en palabras de este mismo autor, "una orientación funcional determinada", comprendiendo cada una de las fases citadas de uno 
a cinco microciclos, variando su número en función de la clase de orientación asignada a la lase en cuestión y la ubicación temporal del macrociclo integrado temporal con respecto a la competición principal del ciclo de preparación (figura 2).

\section{FASES DEL MACROCICLO INTEGRADO}

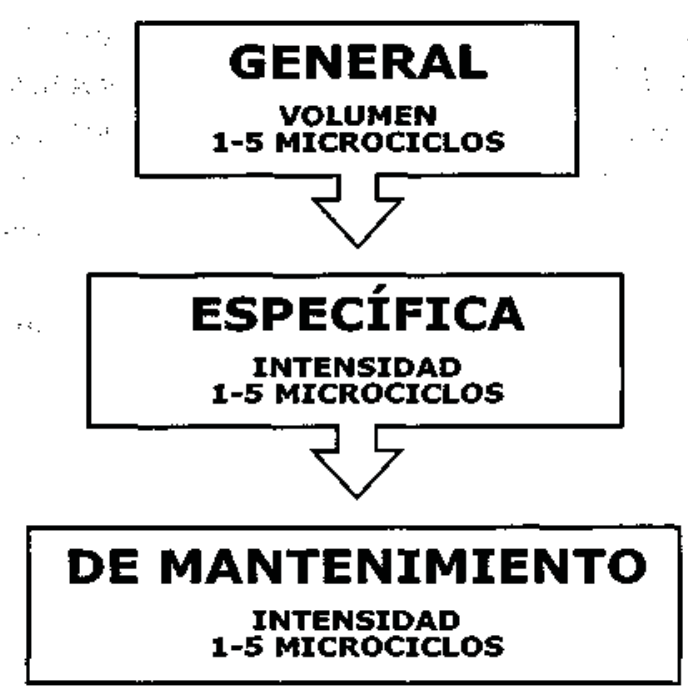

FIG. 2. Estructura del macrociclo integrado. Tomado de Navarro (1998)

\section{EL TRABAJO EN EQUIPO MULTIDISCIPLINAR}

El trabajo del equipo constituido por especialistas incorporaría diferentes etapas (Fuentes, 1999) (cuadral):

1. Asesoramiento y posterior composición de un equipo multidisciplinar que tenga la máxima experiencia en el tratamiento de tenistas.

El entrenamiento integrado y, por lo tanto, el trabajo en equipo y el consenso entre todos los componentes que lo forman aportarán al entrenamiento $y$, consecuentemente, a la competición la mayor calidad posible.

El equipo en cuestión debería estar compuesto por los siguientes especialistas:

- Entrenador: eje del equipo y ejecutor del proceso.

- Ayudante del entrenador.

- Preparador físico.

- Psicólogo.

- Médico.
- Fisioterapeuta.

- Dietista.

- Biomecánico.

- Manager

- Jugador

2. Perfeccionamiento del entrenador por parte del equipo multidisciplinar, y de este último por parte del entrenador.

Será necesario que en el seno del equipo se desarrolle una dinámica de enseñanza-aprendizaje interactiva entre todos los integrantes del equipo: en primer lugar, mediante charlas unipersonales del entrenador con cada uno de los miembros en cuestión, y, en segundo lugar, mediante reuniones en grupo donde se solventen ciertas carencias básicas que presenta cada uno de los componentes del mismo con relación a los contenidos más específicos y aplicables de las diferentes especialidades.

3. Perfeccionamiento de la información, evaluación e interpretación de las características del tenis de alta competición.

Una vez creado el equipo, y tras una visión más global de lo que acontece en el seno del mismo desde el punto de vista físico, psicológico, etc., resultará imprescindible concretar y hacer explícitos cuáles son aquellos rasgos fundamentales que desde las distintas especialidades definen el tenis de alta competición.

4. Estudio y adquisición de los recursos humanos y materiales a los que podemos acceder.

- Respaldo económico.

- Instalaciones y material del que disponemos o que nos pueden ser cedidos.

- Compañeros de entrenamiento: deben ser de un nivel similar y, si es posible, que posean distintos patrones de juego, con el objeto de efectuar unas adaptaciones más flexibles, especialmente porque se efectuará un buen número de entrenamientos modelados, los cuales exigen la participación de uno con el otro, no tanto hacer cubos.

- Posibilidades de desplazamiento.

- Fechas y horarios disponibles por parte de todos los miembros del equipo. 


\section{Presentación del equipo al jugador.}

Con ello se potencia el que exista desde el primer momento una idea de equipo y entre todos los especialistas pueda concienciarse al jugador acerca de la importancia de cada uno de sus cometidos, a : fin de encontrar su máximo rendimiento. Esta presentación persigue, entre otros objetivos, que el jugador complete los cuestionarios con la máxima : motivación y convicción posibles.

6. Extracción de los antecedentes del jugador. Se hace imprescindible efectuar diferentes cuestionarios diseñados por o con la ayuda de cada uno de los especialistas del equipo, a fin de poder encontrar el punto de partida.

7. Diseño de estrategias con la ayuda del psicólogo que permitan contrastar y completar la información obtenida sin perjudicar al jugador : y a su entorno.

Estas estrategias deberían, evidentemente, ser diseñadas con la ayuda del psicólogo y ejecutadas por : el propio entrenador.

8. Comparación y perfeccionamiento de la información obtenida del jugador.

- Comunicación con los anteriores entrenadores acerca de su experiencia con el jugador a todos los niveles: técnico, táctico, físico, psicológico, resultados obtenidos, edad de iniciación, etc.

- Comunicación con los padres, con el objeto de completar y contrastar diferentes aspectos (afectivos, académicos, sociales...).

- Búsqueda de información no extraída mediante los anteriores procedimientos: petición de la clasificación del jugador durante su trayectoria deportiva a la federación correspondiente, tests de condición técnico-tácticos, de condición física, psicológicos, etc., realizados en concentraciones.

9. Evaluación inicial del jugador: Se procederá a efectuar dos evaluaciones: a) una evaluación unilateral, realizada a través de batería de tests y de valoraciones, y b) una evaluación multilateral de los aspectos técnicos, tácticos, físicos y psicológicos.
10. Estudio de las competiciones, exhibiciones y otros compromisos comerciales.

Hay que considerar el número de torneos previstos para su disputa, pudiendo ser, perfectamente, treinta y cinco torneos, disputados a lo largo de once meses al año.

11. Reunión del equipo para el tratamiento global de los cuestionarios, de las evaluaciones, estudio de las competiciones, exhibiciones, etc.

Una vez analizados los datos unilateralmente, será necesario que todo el equipo se reúna para establecer las implicaciones entre unos resultados y otros, con el objeto de proceder a la obtención de una información lo más completa, global y aplicable posible.

12. Determinación y operativización de los objetivos. Considerando todos los puntos anteriormente expuestos, se procederá a la determinación de los objetivos.

13. Diseño de la periodización de la temporada. Se establecerá el correspondiente macrociclo (ciclo anual) parcializado en los correspondientes mesociclos (etapas de 3 a 5 semanas cada uno); éstos, parcializados, a su vez, en microciclos (una semana de duración cada uno), y, finalmente, estos últimos en las correspondientes sesiones de entrenamiento (una o más al día).

14. Diseño de instrumentos que permitan reestructurar -en el caso de ser necesario- con celeridad y acierto los objetivos o entrenamientos previamente establecidos.

Deberíamos construir una serie de instrumentos o correlaciones entre los diferentes acontecimientos que vienen sucediéndose y que se alejan de lo establecido a fin de poder regular dichas modificaciones de lo presupuestado.

15. Presentación al jugador de la forma de trabajo. Se efectuará esta presentación con el fin de que el jugador tenga confianza en él mismo y se sienta especialmente implicado en el proceso, lo que sin duda le motivará especialmente. 
16. Puesta en práctica de la periodización. Ha llegado el momento de ejecutar ahora cada una de las sesiones de entrenamiento diseñadas para el macrociclo en cuestión, con las necesarias y pertinentes reestructuraciones, y partiendo de los instrumentos, previamente diseñados, los cuales facilitan la prontitud y calidad de dichas modificaciones como alternativa a lo previamente estimado.

17. Evaluaciones control. Deberán establecerse una serie de evaluaciones control dentro de la periodización, fijándose su número y fecha de realización. Este hecho no implica que ante una desviación importante de lo presupuestado (muchas derrotas por cansancio, numerosos errores en situaciones de presión, etc.) no puedan efectuarse otras evaluaciones con el objeto de intervenir con prontitud ante cualquier problema que surja.

18. Reajustes en la periodización. Bien como consecuencia de la información extraída por las evaluaciones control, bien por las puntuales, etc.

\section{METODOLOGÍA PARA EL DISEÑO DE LAS SESIONES DE ENTRENAMIENTO INTEGRADO}

A) Respetar los principios fundamentales del entrenamiento: de la unidad funcional, de la individualización, de la transferencia, del incremento paulatino del esfuerzo, etc.

B) Analizar la situación que provoca el golpe propuesto para su entrenamiento.

C) Analizar la situación que se presenta como consecuencia de la ejecución del golpe propuesto para su entrenamiento.

D) Realizar sesiones mixtas en las que se combinen momentos de aprendizaje, de mantenimiento y de perfeccionamiento.

E) Procurar otorgar durante el entrenamiento la mayor autonomía posible al entrenador. El entrenador debe ayudarse de otra persona para el cometido de lanzar las pelotas al jugador, con el objeto de que el primero se encuentre en la mejor posición y concentración posibles que le permitan aportar la máxima calidad a las correcciones, indicaciones, demostraciones, etc.

F) Explicar al alumno el proceso de entrenamiento para que crea en él y también pueda aportar ideas, lo que dará confianza al jugador, que aprenderá a ser más autónomo en sus decisiones en la pista y aumentará su grado de implicación y responsabilidad.

G) Realizar sesiones no demasiado largas.

H) Distribuir correctamente las sesiones de entrenamiento: en caso de efectuar dos sesiones de entrenamiento, especialmente en el período competitivo, las sesiones matinales suelen orientarse hacia objetivos condicionales de alta intensidad, puesto que el descanso nocturno permite la recuperación orgánica del individuo. Las sesiones vespertinas están orientadas hacia objetivos técnicos fundamentalmente, teniendo cabida también los objetivos condicionales a intensidades específicas.

I) Analizar las demandas físicas, técnicas, tácticas y psicológicas con relación a las consideraciones médicas, dietéticas y fisioterapéuticas del jugador tras las correspondientes evaluaciones, para posibilitar la creación de ejercicios lo más aplicados posible a la situación real de juego (escasa potencia de piernas, mala volea baja, ejecución de numerosas dejadas de forma inoportuna ante jugadores con un buen juego de piernas, gran cantidad de dobles faltas en los momentos decisivos del partido por exceso de presión).

J) Cuantificar la magnitud de las cargas de trabajo (duración, repeticiones, series, pausas e intensidad) necesarias a nivel físico, tipo de ejercicio, volumen, intensidad.

K) Cuantificar la magnitud de las cargas de trabajo (duración, repeticiones, series, pausas e intensidad) necesarias a nivel técnico.

L) Cuantificar la magnitud de las cargas de trabajo (duración, repeticiones, series, pausas e intensidad) necesarias a nivel táctico. 
comete numerosas dobles faltas en los momentos

M) Cuantificar la magnitud de las cargas de trabajo (duración, repeticiones, series, pausas e intensidad) necesarias a nivel psicológico.

N) Cuantificar (alimentos, cantidad, momento, forma...) las necesidades alimentarias.

N) Cuantificar las necesidades fisioterapéuticas (tipo de prevenciones, momento de empleo de las mismas, ejercicios de compensación necesarios, regeneración tras el entrenamiento -masaje, sauna, carrera, estiramientos...-).

O) Cuantificar las necesidades médicas (prescripciones médicas, primeros auxilios...).

P) Emplear la practica imaginada cuando el jugador se encuentra lesionado, ya que puede entrenar psicológicamente sus técnicas deportivas, favoreciendo el recuerdo de las mismas, a la par que incrementa su autoconfianza y motivación hacia su vuelta al entrenamiento en condiciones reales.

Q) Emplear la biomecánica para las correcciones. Según Dent y Pankhurst (1998), "La técnica óptima puede definirse como aquella que permite la combinación más eficiente de potencia y control tanto en el golpe como en el movimiento al tiempo que reduce el riesgo de lesión. A la hora de analizar y mejorar la técnica avanzada, un buen conocimiento de la biomecánica ayudará a los entrenadores a evitar fijarse en los aspectos "cosméticos" del golpe (por ejemplo: su apariencia) y centrarse en su efectividad".

R) La integración de los elementos (cuadro 2).

-Elementos técnicos con elementos tácticos. Ejemplo: Entrenamiento de la dejada tras la provocación del alejamiento del jugador contrario de la línea de fondo.

-Elementos técnicos con elementos físicos. Ejemplo: Entrenamiento del gesto técnico y de la regulación de la potencia de la derecha liftada y plana en posición abierta ante situaciones de emergencia de golpeo de derecha.

-Elementos técnicos con elementos psicológicos. Ejemplo: Entrenamiento del servicio y de la concentración ante situaciones en las que el jugador decisivos del partido.

-Elementos tácticos con elementos físicos. Ejemplo: Entrenamiento de la primera volea tarde tras la ejecución de un primer servicio con subida a la red.

-Elementos tácticos con elementos psicológicos. Ejemplo: Entrenamiento de la adopción de diferentes planteamientos tácticos en función de las condiciones del partido y de las características del jugador rival.

- Elementos físicos con elementos psicológicos. Ejemplo: Entrenar la subida a la red tras el servicio en condiciones de fatiga, entrenar la visualización de las distintas opciones básicas que pueden darse tras la subida a la red.

S) Hacia la integración del mayor número de elementos posible.

\section{EJEMPLO DEL ÚLTIMO EJERCICIO TÉCNICO-TÁCTICO DE UNA SESIÓN DE ENTRENAMIENTO INTEGRADO}

Presentamos a continuación un ejemplo de ejercicio técnico-táctico correspondiente a una sesión de entrenamiento integrado en el tenis (Fuentes, 2000).

\section{DENOMINACIÓN.}

"Partido en condiciones de juego modificadas. El resto ante un jugador de fondo". Localizarían de la sesión:

Dentro del período precompetitivo.

Duración: Disputa de un set.

Objetivo Específico.

Mejorar la ejecución del resto como golpe de construcción (ni defensivo ni ganador) ante el segundo servicio de un jugador de fondo (sólido y defensivo).

\section{Descripción:}

Tan solo existirán segundos servicios; si un jugador no introduce su único servicio, pierde dos puntos (030 ), puesto que, en teoría, un jugador de fondo no debe tener un segundo servicio muy débil (para evitar que pueda entrarse en una dinámica para él muy favorable de "peloteo"), ya que esta debilidad en el segundo servicio facilitaría la subida a la red del jugador contrario; si introduce el único servicio de que dispone y el jugador que resta lanza la pelota fuera de los límites de la pista de individuales, perderá 2 puntos 
(30-0) (para evitar que en vez de emplear golpes de construcción procure ganar el punto de forma directa. No es ese nuestro objetivo); si el jugador que efectúa el servicio gana el punto durante la disputa del mismo de manera ofensiva tras el resto (no manteniéndose en el fondo de la pista, o efectuando un golpe ganador desde el fondo de la misma), perderá un juego completo (0-1). Si el jugador que resta gana el punto tras un golpe efectuado inmediatamente después del resto de construcción, ganará 3 puntos (0-40). El resto de puntos ganados mediante cualquier otra situación o golpe valdrá 1 punto (15-0 o 0-15), no pudiéndose acumular puntos para el siguiente juego.

\section{CONCLUSIONES}

El conocimiento de los procedimientos para desarrollar el entrenamiento integrado en la alta competición ofrece al entrenador una visión de conjunto del tenis donde lo técnico, lo táctico, lo físico y lo psicológico no se tratan como compartimentos estancos, sino como lo que son: un conjunto de interacciones de diversos matices que conforman el comportamiento humano y, por ende, el de todo jugador de tenis de alta competición.

En nuestra opinión, el entrenador de alta competición, así como el entrenador de jugadores de otros niveles de juego inferiores, ha de procurar cuantificar el grado de implicación de cada uno de los aspectos con los restantes en sus sesiones, con el objeto de trabajar de la forma más eficaz posible: restando minutos de entrenamiento innecesarios, disminuyendo las posibilidades de que se lleve al jugador al sobreentrenamiento, reduciendo las posibilidades de que se produzcan lesiones.. .Y que el jugador pueda entrenarse de la forma lo mas parecida posible a la situación de partido, haciendo hincapié, mediante la modificación de la situación real de juego, en aquellos aspectos que nos interese trabajar entrenar.

\section{BIBLIOGRAFIA}

ALVARO, J. (1992) "Preparación Física Específica, en W. AA, Balonmano, Madrid, Comité Olímpico Español - Real Federación Española de Balonmano, pp. 291-377.
BALAGUER, I. (1996) "Entrenamiento psicológico en un grupo de competición femenino de tenis", Apunts: Educación Física y Deportes, n. ${ }^{\circ} 44-45$ (Barcelona), pp. 143-153. DENT, P.; PANKHURST, A. (1998) "Análisis técnico: Biomecánica práctica", ITF Coaches Review, $6^{\circ}$ año, n. ${ }^{\circ} 14$, abril, número especial: $10^{\circ}$ Seminario Mundial de Entrenadores de la ITF (cont.), pp. 46. FUENTES, J. P. (1997) "Entrenamiento visual y rendimiento tenístico", Ponencia presentada en el ICongreso Hispano-Portugués de Tenis, Cáceres, 9, 10 y 11 de diciembre de 1997. FUENTES,J.P.(1999)

"Entrenamiento integral en el tenis de competición", en D.Sanz,yP.del Río (coords.) Actas del Congreso Nacional de $T$ e n i s : "Entrenamiento en alto rendimiento", Universidad de La Rioja, Área Docente de la Real F ederación Española de Tenis (ENMT), pp. 2338.

\section{FUENTES,J.P.(2000)}

$$
\begin{aligned}
& \text { El conocimiento de los } \\
& \text { procedimientos para desarrollar } \\
& \text { el entrenamiento integrado en } \\
& \text { la alta competición ofrece al } \\
& \text { entrenador una visión de } \\
& \text { conjunto del tenis donde lo } \\
& \text { técnico, lo táctico, lo fisico y lo } \\
& \text { psicológico no se tratan como } \\
& \text { compartimentos estancos, sino } \\
& \text { como lo que son-, un conjunto de } \\
& \text { interacciones de diversos matices } \\
& \text { que conforman el } \\
& \text { comportamiento humano y, por } \\
& \text { ende, el de todo jugador de tenis } \\
& \text { de alta competición. }
\end{aligned}
$$
en el tenis", en J. A. García; J. S. Damas; J. P. Fuentes, Entrenamiento en balonmano, voleibol y tenis, Universidad de Extremadura, Departamento de Didáctica de la Expresión Musical, Plástica y Corporal, Instituto de Ciencias de la Educación, Universitas editorial, pp. 241-300, esp. pp. 265-289. GARCÍA, J. A. (1997/1998) El entrenamiento integrado

en balonmano para las categorias de cadetes y juveniles. Comunicaciones técnicas, $\mathrm{n} .{ }^{\circ} 4$, Federación de Castilla y León de Balonmano, Escuela Territorial de Entrenadores. 
Simposium F. Europe/F. Internacional. Garmish 89, Madrid, Real Federación Española de Tenis, Escuela Nacional de Maestría de Tenis, pp. 24-28.

LORENZO, M. (1988) "Operativización de objetivos", en W. AA., Didáctica general, Madrid, Anaya (Textos Universitarios), pp. 85-107.

LOTH, J. P. (1984) "El entrenamiento en el tenis", en J. P. Cousteau, Medicina del tenis, Barcelona, Masson, pp. 8-12.

MARGETS, A. (1996) "Planificación del entrenamiento: Las diferentes etapas del tenista", Apunts: Educación Física y Deportes, n. ${ }^{\circ} 44-45$ (Barcelona), pp. 81-86.

MOURE, T. (1996) "Planificación del entrenamiento", Apunts: Educación Física y Deportes, n. ${ }^{\circ} 44-45$ (Barcelona), pp. 87-93.

NAVARRO, F. (1998) La resistencia en el deporte, Madrid, Gymnos, 1998.

NICK BOLLETTIERI INTERNATIONAL COACHES ASSOCIATION (1997) "Periodización", España, Club Las Lomas, Madrid, abril.

PÉREZ, G. (1996) "Aplicaciones de la psicología al tenis de alto nivel", Apunts-. Educación Física y Deportes, n. ${ }^{\circ} 44-45$ (Barcelona), pp. 136-142.

RASICCI, A. (1986) "Entrenamiento modelado", en/ Simposium Int. Prof. Enseñanza. Barcelona 1986, Madrid, Real Federación Española de Tenis, Escuela Nacional de Maestría de Tenis (s. pág.).

SCHÖNBORN, R. (1983) "Planificación del entrenamiento", en Federación Europea de Tenis (ETA), Montreux. 1983, Madrid, Real Federación
Española de Tenis, Escuela Nacional de Maestría de Tenis, pp. 1-22.

- (1987) "Aproximación científica al tenis. Reglas para el entrenamiento moderno", en $V$

International Tennis Federation Worlwide Coaches Workshop and European Tennis Association Coaches Symposium. Mallorca, Madrid, Real Federación Española de Tenis, Escuela Nacional de Maestría de Tenis.

- (1989) "Nuevos test y estudios sobre el entrenamiento en tenis: principios fisiológicos", en $N$ Simposium Int. Prof. Enseñanza. Granada, 1989, Madrid, Real Federación Española de Tenis, Escuela Nacional de Maestría de Tenis, pp. 223239.

- SINGER, R. N.i y OTROS (1994) "Training mental quickness in beginning/intermediate tennis player", Sport psychologist- 8, n. ${ }^{\circ} 3$, pp. 305-318.

" SOLANELLAS, F. (1998) "Biomecánica", en Curso de Profesor Nacional de Tenis, Madrid, Real

Federación Española de Tenis. Escuela Nacional de Maestría de Tenis.

: STOJAN, S. (1988) "Ejercicios de entrenamiento modelado", en Simposium Europ. Tennis Assoc. (ETA). Madeira, 1988, Madrid, Real Federación

- $\quad$ Española de Tenis, Escuela Nacional de Maestría - de Tenis, pp. 26-36.

- TORRESCUSA, L. C. (1992) "Metodología de la : enseñanza", en W. AA., Balonmano, Madrid, Comité : Olímpico Español, Real Federación Española de - Balonmano, pp. 163-289. 
CUADRO 1. Etapas del trabajo en equipo multidisciplinar en el tenis de alta competición

\section{EQUIPO MULTIDISCIPLINAR}

ETAPAS DEL TRABAJO

1. Asesoramiento $\mathrm{Y}$ posterior composición de un equipo multidisciplinar que tenga la máxima experiencia en el tratamiento de tensitas.

2. Perfeccionamiento del entrenador por parte del equipo multidisciplinar, y de este último parte del entrenador.

3. Perfeccionamiento de la información, evaluación e interpretación de las características del tenis de alta competición.

4. Estudio y adquisición de los recursos humanos y materiales a los que podemos acceder.

5. Presentación del equipo al jugador.

6. Extracción de los antecedentes del jugador.

7. Diseño de estrategias con la ayuda del psicólogo que permitan contrastar y completar la información obtenida sin perjudicar al jugador y a su entono.

8. Comparación y perfeccionamiento de la información obtenida del jugador.

9. Evaluación inicial del jugador.

10. Estudio de las competiciones, exhibiciones y otros compromisos comerciales.

11. Reunión del equipo para el tratamiento global de los cuestionarios, de las evaluaciones, estudio de las competiciones, exhibiciones, etc.

12. Determinación y operativización de los objetivos.

13. Diseño de la periodización de la temporada.

14 Diseño de instrumentos que permitan reestructurar -en el caso de ser necesario-con celeridad y acierto los objetivos o entrenamientos previamente establecidos.

15. Presentación al jugador de la forma de trabajo.

16. Puesta en práctica de la periodización.

17. Evaluaciones control.

18. Reajustes en la periodización. 
CUADRO 2. Metodología para el diseño de las sesiones de entrenamiento integrado: la integración de los elementos

\begin{tabular}{|c|l|}
\hline \multicolumn{2}{|c|}{ ENTRENAMIENTO INTEGRADO } \\
\hline \multicolumn{2}{|c|}{ LA INTEGRACIÓN DE LOS ELEMENTOS EN LAS SESIONES } \\
\hline ELEMENTOS Elementos técnicos con elementos tácticos & $\begin{array}{l}\text { Entrenamiento de la dejada tras la provocación } \\
\text { del alejamiento del jugador contrario de la línea } \\
\text { de fondo }\end{array}$ \\
\hline - Elementos técnicos con elementos físicos & $\begin{array}{l}\text { Entrenamiento del gesto técnico y de la regulación } \\
\text { de la potencia de la derecha liftada y plana en } \\
\text { posición abierta ante situaciones de emergencia } \\
\text { de golpeo de derecha }\end{array}$ \\
\hline - Elementos técnicos con elementos psicológicos & $\begin{array}{l}\text { Entrenamiento del servicio y de la concentración } \\
\text { ante situaciones en las que el jugador comete } \\
\text { numerosas dobles faltas en los momentos decisivos } \\
\text { del partido }\end{array}$ \\
\hline$\cdots$ & $\begin{array}{l}\text { Entrenamiento de la primera volea tarde tras la } \\
\text { ejecución de un primer servicio con subida a la } \\
\text { red }\end{array}$ \\
\hline - Elementos tácticos con elementos psicológicos & $\begin{array}{l}\text { Entrenamiento de la adopción de diferentes } \\
\text { planteamientos tácticos en función de las } \\
\text { condiciones del partido y de las características del } \\
\text { jugador rival }\end{array}$ \\
\hline \multirow{2}{*}{ Elementos tácticos con elementos físicon elementos psicológicos } & $\begin{array}{l}\text { Entrenar la subida a la red tras el servicio en } \\
\text { condiciones de fatiga, entrenar la visualización de } \\
\text { las distintas opciones básicas que pueden darse } \\
\text { tras la subida a la red }\end{array}$ \\
\hline
\end{tabular}

